\title{
Crystal structure of catena-poly[(1,4-diazabicyclo[2.2.2]octane)tetrakis(2- thiopheneacetato)dicopper(II)], $\mathrm{Cu}_{2}\left(\mathrm{C}_{6} \mathrm{H}_{12} \mathrm{~N}_{2}\right)\left(\mathrm{C}_{6} \mathrm{H}_{5} \mathrm{O}_{2} \mathrm{~S}\right)_{4}$
}

\author{
Y.-L. Zhou ${ }^{\mathrm{l}}$, H.-Y. He' and L.-G. Zhu*.I \\ 1 Jiaxing College, Department of Chemical Engineering, Jiaxing, 314001 P. R. China \\ II Zhejiang University, Department of Chemistry, Hangzhou, 310027 P. R. China
}

Received May 19, 2005, accepted and available on-line July 9, 2005; CCDC no. 1267/1554

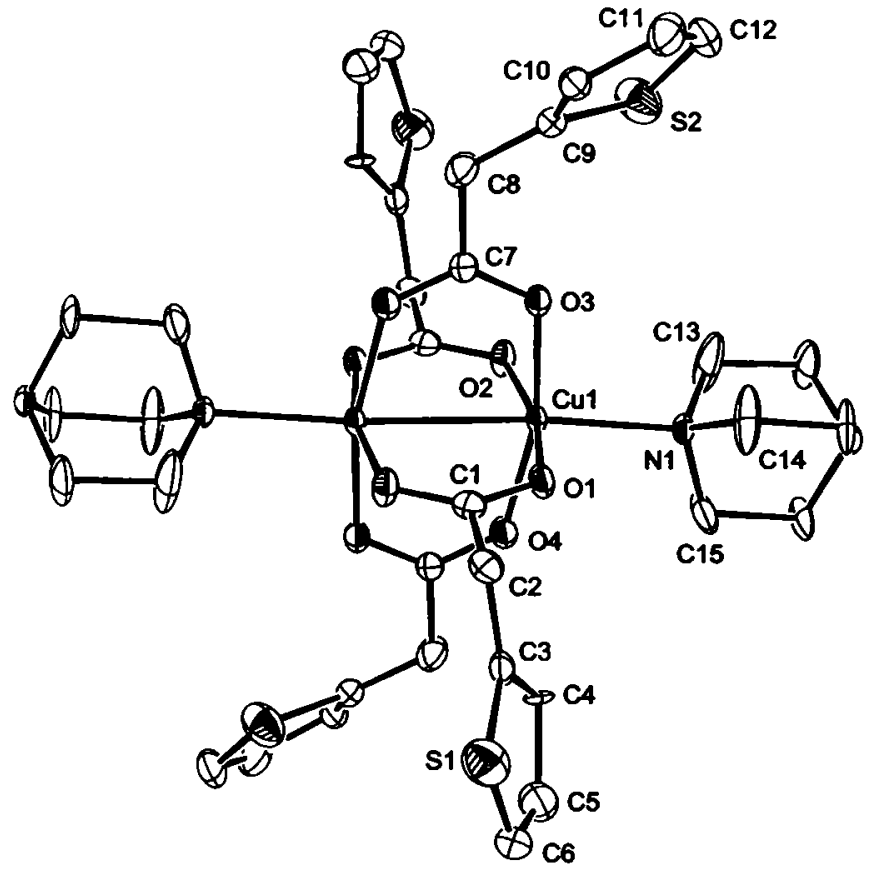

Abstract

$\mathrm{C}_{30} \mathrm{H}_{32} \mathrm{Cu}_{2} \mathrm{~N}_{2} \mathrm{O}_{8} \mathrm{~S}_{4}$, monoclinic, $P 12, / n 1$ (no. 14), $a=9.7712(9) \AA, b=10.734(1) \AA, c=15.457(2) \AA$, $\beta=98.136(2)^{\circ}, V=1604.9 \AA^{3}, Z=2, R_{\mathrm{gt}}(F)=0.061$, $w R_{\text {ref }}\left(F^{2}\right)=0.191, T=298 \mathrm{~K}$.

\section{Source of material}

The complex was synthesized by layer-method using three-layer solutions in a slender tube. The upper layer solution was $5 \mathrm{ml}$ of $\mathrm{CH}_{3} \mathrm{OH}$ containing $0.06 \mathrm{M}$ 1,4-diazabicyclo[2.2.2] octane. The bottom layer solution was $5 \mathrm{ml}$ of DMF solution containing $0.03 \mathrm{M} \mathrm{Cu}\left(\mathrm{NO}_{3}\right)_{2} \cdot 3 \mathrm{H}_{2} \mathrm{O}$ and $0.06 \mathrm{M} 2$-thiopheneacetic acid. The intermediate layer was $5 \mathrm{ml}$ of $\mathrm{CH}_{3} \mathrm{OH} / \mathrm{DMF}(v / v 1: 1)$ mixed solvent. After standing for two weeks, green block-shaped crystals were obtained and collected by suction filtration.

\section{Experimental details}

The carbon-bonded $\mathrm{H}$ atoms were generated geometrically $\left(d\left(\mathrm{C}_{\text {aromatic }}-\mathrm{H}\right)=0.93 \AA, d\left(\mathrm{C}_{s p 3}-\mathrm{H}\right)=0.97 \AA, U_{\text {iso }}=1.2 U_{\mathrm{eq}}\right)$. These were included in the refinement in the riding model approximation. The 1,4-diazabicyclo[2.2.2] octane ligand is in disorder created by the inversion center and considered over two sites. The small $U$ values of C4 may be caused by a little part of disordered sulfur atoms which occupy the $\mathrm{C} 4$ position.

\section{Discussion}

Thiophene derivatives show biological activities and have been used in pharmaceutical and clinical purpose [1]. Recently, thiophenecarboxylate complexes have received increasing attention, but only seven 2-thiopheneacetate (tpa) complexes have been structurally characterized [1-4]. Two copper complexes of them have a dinuclear $\left[\mathrm{Cu}_{2}(\mathrm{COO})_{4}\right]$ unit, which has been widely reported in copper(II) carboxylates due to interests in exchangecoupling interactions and special bioactive and biocatalytic functions in the past decades. Herein we present a new 1D 2thiopheneacetate copper(II) complex containing a dinuclear [ $\left.\mathrm{Cu}_{2}(\mathrm{COO})_{4}\right]$ motif.

The complex is a one-dimensional chain containing a dinuclear $\left[\mathrm{Cu}_{2}(\mathrm{COO})_{4}\right]$ unit, in which each copper(II) atom is coordinated by four oxygen donors of four carboxylate groups from four 2thiopheneacetato ligands acting in a syn,syn bridging mode. The apical position of the copper(II) coordination polyhedron (along the $\mathrm{Cu}-\mathrm{Cu}$ direction) is occupied by nitrogen atom from the 1,4-diazabicyclo[2.2.2]octane (dbco) ligand. Each dbco bridges two dinuclear $\left[\mathrm{Cu}_{2}(\mathrm{COO})_{4}\right]$ units and extends the structure into a one-dimensional chain. In the dinuclear $\left[\mathrm{Cu}_{2}(\mathrm{COO})_{4}\right]$ motif, the $\mathrm{Cu}-\mathrm{O}$ distances are similar to those of the complex $\left[\mathrm{Cu}(\mathrm{tpa})_{2}(\mathrm{DMF})\right]_{2}[3]$, but is very different from the complex $\left[\mathrm{Cu}_{2} \text { (tpa) }\right]_{n}[1]$. The $\mathrm{Cu}-\mathrm{N}$ distance in the title complex is significant longer than $\mathrm{Cu}-\mathrm{O}$ bond distances due to the apical coordination of the $\mathrm{dbco}$ ligand. The $\mathrm{Cu}-\mathrm{Cu}$ distance in the dimeric units depends on the bridging and apical ligands. The separation of $2.699(1) \AA$ in the title complex is longer than those of $\left[\mathrm{Cu}(\mathrm{tpa})_{2}(\mathrm{DMF})_{2}\right]_{2}$ and $\left[\mathrm{Cu}_{2}(\mathrm{tpa})_{4}\right]_{n}$ or dinuclear copper acetates, indicating the dbco linker results in a weaker $\mathrm{Cu}$...Cu interaction. As a consequence of such interaction, the $\mathrm{O}-\mathrm{C}-\mathrm{O}$ angles in the $\mathrm{Cu}-\mathrm{O}-\mathrm{C}-\mathrm{O}-\mathrm{Cu}$ rings are greater than those of $\left[\mathrm{Cu}_{2}(\mathrm{tpa})_{4}\right]_{n}$ and $\left[\mathrm{Cu}(\text { tpa })_{2}(\mathrm{DMF})_{2}\right]_{2}$.

Table 1. Data collection and handling.

\begin{tabular}{|c|c|}
\hline $\begin{array}{l}\text { Crystal: } \\
\text { Wavelength: }\end{array}$ & $\begin{array}{l}\text { green block, size } 0.12 \times 0.15 \times 0.21 \mathrm{~mm} \\
\text { Mo } K_{a} \text { radiation }(0.71073 \AA) \\
16.39 \mathrm{~cm}^{-1}\end{array}$ \\
\hline $\begin{array}{l}\mu \text { : } \\
\text { Diffractometer, scan mode: }\end{array}$ & $\begin{array}{l}10.39 \mathrm{~cm} \\
\text { Bnker SMART APEX CCD } 0 / 0\end{array}$ \\
\hline $2 \theta_{\max }$ : & $50^{\circ}$ \\
\hline$N(\overline{h k l})_{\text {measured, }} N(\boldsymbol{h k l})_{\text {unique: }}$ & 7941,2816 \\
\hline Criterion for $I_{\text {obs, }} N(h k l)_{g t:}$ & $I_{\text {obs }}>2 \sigma\left(I_{\text {obs }}\right), 2122$ \\
\hline$N$ (param) hefined: & 236 \\
\hline Programs: & $\begin{array}{l}\text { SHELXXS-97 [5], SHELX]-97 [6], } \\
\text { ORTEP-3 [7], WinGX [8] }\end{array}$ \\
\hline
\end{tabular}

* Correspondence author (e-mail: chezlg@ zju.edu.cn) 
Table 2. Atomic coordinates and displacement parameters (in $\AA^{2}$ ).

\begin{tabular}{lllllll}
\hline Atom & Site & Occ & \multicolumn{1}{l}{$\boldsymbol{x}$} & \multicolumn{1}{l}{$y$} & \multicolumn{1}{l}{$U_{\text {iso }}$} \\
\hline H(2A) & $4 e$ & & 0.6242 & 0.2258 & 1.2426 & 0.044 \\
H(2B) & $4 e$ & & 0.4705 & 0.1951 & 1.2539 & 0.044 \\
H(4) & $4 e$ & & 0.7091 & -0.0832 & 1.2345 & 0.022 \\
H(5) & $4 e$ & & 0.7436 & -0.1983 & 1.3811 & 0.070 \\
H(6) & $4 e$ & & 0.6695 & -0.0887 & 1.4975 & 0.060 \\
H(8A) & $4 e$ & & 0.3986 & 0.3081 & 0.8164 & 0.061 \\
H(8B) & $4 e$ & & 0.3794 & 0.3748 & 0.9034 & 0.061 \\
H(10) & $4 e$ & & 0.5068 & 0.5807 & 0.9212 & 0.050 \\
H(11) & $4 e$ & & 0.7059 & 0.6845 & 0.8715 & 0.078 \\
H(12) & $4 e$ & & 0.8296 & 0.5518 & 0.7878 & 0.094 \\
H(3E) & $4 e$ & 0.5 & 0.8604 & 0.0605 & 0.8764 & 0.050 \\
\hline
\end{tabular}

Table 2. Continued.

\begin{tabular}{|c|c|c|c|c|c|c|}
\hline Atom & Site & Occ. & $x$ & $y$ & $z$ & $U_{\text {iso }}$ \\
\hline$H(3 F)$ & $4 e$ & 0.5 & 0.8672 & -0.0847 & 0.8867 & 0.05 \\
\hline$H(4 E)$ & $4 e$ & 0.5 & 0.9181 & 0.1227 & 1.1000 & 0.05 \\
\hline$H(4 F)$ & $4 e$ & 0.5 & 0.9012 & 0.1887 & 1.0086 & 0.05 \\
\hline H(5E) & $4 e$ & 0.5 & 0.8855 & -0.1772 & 1.0269 & 0.05 \\
\hline$H(5 F)$ & $4 e$ & 0.5 & 0.9136 & -0.0929 & 1.1104 & 0.05 \\
\hline $\mathbf{H}\left(3 \mathrm{E}^{\prime}\right)$ & $4 e$ & 0.5 & 0.8933 & 0.1875 & 0.9807 & 0.05 \\
\hline$H\left(3 F^{\prime}\right)$ & $4 e$ & 0.5 & 0.8648 & 0.1115 & 0.8932 & 0.05 \\
\hline$H\left(4 E^{\prime}\right)$ & $4 e$ & 0.5 & 0.9166 & -0.0720 & 1.1187 & 0.05 \\
\hline $\mathbf{H}\left(\mathbf{4 F ^ { \prime }}\right)$ & $4 e$ & 0.5 & 0.9128 & 0.0743 & 1.1210 & 0.05 \\
\hline $\mathbf{H}\left(5 \mathrm{E}^{\prime}\right)$ & $4 e$ & 0.5 & 0.8617 & -0.1136 & 0.8992 & 0.05 \\
\hline$H\left(5 F^{\prime}\right)$ & $4 e$ & 0.5 & 0.8783 & -0.1814 & 0.9900 & 0.05 \\
\hline
\end{tabular}

Table 3. Atomic coordinates and displacement parameters (in $\AA^{2}$ ).

\begin{tabular}{|c|c|c|c|c|c|c|c|c|c|c|c|}
\hline Atom & Site & Occ. & $x$ & $y$ & $z$ & $U_{11}$ & $U_{22}$ & $U_{33}$ & $U_{12}$ & $U_{13}$ & $U_{23}$ \\
\hline $\mathrm{Cu}(1)$ & $4 e$ & & $0.63731(6)$ & $0.00727(5)$ & $0.99762(4)$ & $0.0146(4)$ & $0.0319(5)$ & $0.0342(5)$ & $-0.0005(3)$ & $0.0057(3)$ & $0.0035(3)$ \\
\hline$S(1)$ & $4 e$ & & $0.5902(2)$ & $0.0790(2)$ & $1.4117(1)$ & $0.077(2)$ & $0.076(2)$ & $0.067(1)$ & $-0.005(1)$ & $0.020(1)$ & $-0.002(1)$ \\
\hline$S(2)$ & $4 e$ & & $0.6923(2)$ & $0.3796(2)$ & $0.8067(2)$ & $0.074(1)$ & $0.071(1)$ & $0.086(2)$ & $0.010(1)$ & $0.041(1)$ & $0.005(1)$ \\
\hline$O(1)$ & $4 e$ & & $0.6380(4)$ & $0.0923(4)$ & $1.1109(2)$ & $0.024(2)$ & $0.050(3)$ & $0.035(2)$ & $-0.007(2)$ & $0.010(2)$ & $-0.003(2)$ \\
\hline$O(2)$ & $4 e$ & & $0.5901(4)$ & $-0.0757(4)$ & $0.8830(2)$ & $0.022(2)$ & $0.055(3)$ & $0.041(2)$ & $-0.001(2)$ & $0.006(2)$ & $-0.010(2)$ \\
\hline$O(3)$ & $4 e$ & & $0.5987(4)$ & $0.1701(4)$ & $0.9391(3)$ & $0.023(2)$ & $0.039(2)$ & $0.060(3)$ & $-0.001(2)$ & $0.004(2)$ & $0.017(2)$ \\
\hline$O(4)$ & $4 e$ & & $0.6262(4)$ & $-0.1547(4)$ & $1.0566(3)$ & $0.027(2)$ & $0.038(2)$ & $0.059(3)$ & $0.001(2)$ & $0.008(2)$ & $0.014(2)$ \\
\hline$C(1)$ & $4 e$ & & $0.5317(6)$ & $0.1069(5)$ & $1.1479(4)$ & $0.039(4)$ & $0.030(3)$ & $0.034(3)$ & $0.001(3)$ & $0.006(3)$ & $0.005(2)$ \\
\hline$C(2)$ & $4 e$ & & $0.5558(6)$ & $0.1598(5)$ & $1.2395(4)$ & $0.039(3)$ & $0.034(3)$ & $0.039(3)$ & $0.000(3)$ & $0.012(3)$ & $-0.002(2)$ \\
\hline$C(3)$ & $4 e$ & & $0.6059(5)$ & $0.0582(5)$ & $1.3045(3)$ & $0.023(3)$ & $0.053(4)$ & $0.032(3)$ & $-0.011(3)$ & $0.004(2)$ & $-0.003(3)$ \\
\hline $\mathrm{C}(4)$ & $4 e$ & & $0.6817(5)$ & $-0.0569(4)$ & $1.2868(3)$ & $0.034(3)$ & $0.012(2)$ & $0.008(2)$ & $-0.002(2)$ & $-0.006(2)$ & $0.003(2)$ \\
\hline$C(5)$ & $4 e$ & & $0.7029(7)$ & $-0.1199(6)$ & $1.3736(4)$ & $0.051(4)$ & $0.054(4)$ & $0.070(5)$ & $0.007(3)$ & $0.015(4)$ & $0.015(4)$ \\
\hline$C(6)$ & $4 e$ & & $0.6604(7)$ & $-0.0579(6)$ & $1.4407(4)$ & $0.045(4)$ & $0.064(4)$ & $0.039(4)$ & $-0.005(3)$ & $0.007(3)$ & $0.012(3)$ \\
\hline$C(7)$ & $4 e$ & & $0.4773(6)$ & $0.2080(5)$ & $0.9236(3)$ & $0.029(3)$ & $0.034(3)$ & $0.034(3)$ & $-0.004(2)$ & $0.002(2)$ & $0.002(2)$ \\
\hline$C(8)$ & $4 e$ & & $0.4454(6)$ & $0.3295(6)$ & $0.8741(5)$ & $0.035(4)$ & $0.043(4)$ & $0.073(5)$ & $0.004(3)$ & $-0.003(3)$ & $0.016(3)$ \\
\hline$C(9)$ & $4 e$ & & $0.5587(5)$ & $0.4173(5)$ & $0.8620(4)$ & $0.029(3)$ & $0.041(3)$ & $0.041(3)$ & $0.001(3)$ & $0.003(2)$ & $0.011(3)$ \\
\hline$C(10)$ & $4 e$ & & $0.5679(6)$ & $0.5420(5)$ & $0.8887(4)$ & $0.031(3)$ & $0.038(3)$ & $0.057(4)$ & $0.006(3)$ & $0.010(3)$ & $0.014(3)$ \\
\hline$C(11)$ & $4 e$ & & $0.6826(7)$ & $0.6016(7)$ & $0.8597(5)$ & $0.047(4)$ & $0.043(4)$ & $0.098(6)$ & $-0.007(3)$ & $-0.011(4)$ & $0.011(4)$ \\
\hline$C(12)$ & $4 e$ & & $0.7539(8)$ & $0.5260(7)$ & $0.8135(6)$ & $0.039(4)$ & $0.088(6)$ & $0.110(7)$ & $-0.005(4)$ & $0.019(5)$ & $0.052(5)$ \\
\hline$N(1)$ & $4 e$ & 0.5 & $0.8663(4)$ & $0.0042(4)$ & $1.0002(3)$ & $0.016(3)$ & $0.030(3)$ & $0.039(3)$ & $-0.001(2)$ & $0.005(2)$ & $0.000(2)$ \\
\hline$C(13)$ & $4 e$ & 0.5 & $0.904(1)$ & $-0.0068(8)$ & $0.9124(9)$ & $0.022(9)$ & $0.23(5)$ & $0.06(1)$ & $0.01(1)$ & $0.008(8)$ & $-0.03(2)$ \\
\hline$C(14)$ & $4 e$ & 0.5 & $0.939(1)$ & $0.115(1)$ & $1.0413(6)$ & $0.019(8)$ & $0.08(1)$ & $0.16(3)$ & $0.003(8)$ & $0.03(1)$ & $-0.05(2)$ \\
\hline$C(15)$ & $4 e$ & 0.5 & $0.928(2)$ & $-0.099(1)$ & $1.0498(6)$ & $0.026(8)$ & $0.09(2)$ & $0.18(4)$ & $0.003(9)$ & $0.02(1)$ & $0.09(2)$ \\
\hline$N\left(1^{\prime}\right)$ & $4 e$ & 0.5 & $0.8663(4)$ & $0.0042(4)$ & $1.0002(3)$ & $0.016(3)$ & $0.030(3)$ & $0.039(3)$ & $-0.001(2)$ & $0.005(2)$ & $0.000(2)$ \\
\hline$C\left(13^{\prime}\right)$ & $4 e$ & 0.5 & $0.913(1)$ & $0.1092(9)$ & $0.9525(6)$ & $0.013(7)$ & $0.05(1)$ & $0.16(3)$ & $0.014(7)$ & $0.02(1)$ & $0.06(2)$ \\
\hline$C\left(14^{\prime}\right)$ & $4 e$ & 0.5 & $0.940(2)$ & $0.0032(8)$ & $1.0892(8)$ & $0.022(8)$ & $0.16(3)$ & $0.046(9)$ & $-0.01(1)$ & $0.015(6)$ & $-0.01(1)$ \\
\hline$C\left(15^{\prime}\right)$ & $4 e$ & 0.5 & $0.908(1)$ & $-0.109(1)$ & $0.9587(6)$ & $0.013(6)$ & $0.048(9)$ & $0.11(2)$ & $-0.009(6)$ & $0.013(8)$ & $-0.03(1)$ \\
\hline
\end{tabular}

Acknowledgment. The project was supported by the National Natural Science Foundation of China (grant no. 50073019).

\section{References}

1. Drozdzewski,P.; Brozyna, A.; Kubiak, M.: Synthesis, X-ray crystal structure and vibrational spectra of a polymeric copper(II) complex with 2thiopheneacetic acid. Polyhedron 23 (2004) 1785-1792.

2. Cai, L. Z.; Chen, W. T.; Wang, M. S.; Guo, G. C.; Huang, J. S.: Syntheses, structures and luminescent properties of four new ID lanthanide complexes with 2-thiopheneacetic acid ligand. Inorg. Chem. Commun. 7 (2004) 611-613.

3. Drozdzewski, P.; Brozyna, A.; Kubiak, M.: New binuclear copper(II) with 2-thiopheneacetic acid. J. Mol. Struct. 707 (2004) 131-137.

4. Koshima, H.; Honke, S.: Chiral bimolecular crystallization of tryptamine and achiral carboxylic acids. J. Org. Chem. 64 (1999) $790-793$.
5. Sheldrick, G. M.: SHELXS-97. Program for the Solution of Crystal Structures. University of Göttingen, Germany 1997.

6. Sheldrick, G. M.: SHELXI-97. Program for the Refinement of Crystal Structures. University of Göttingen, Germany 1997

7. Farrugia, L. J.: ORTEP-3 for Windows - a version of ORTEP-III with a Graphical User Interface (GUI). J. Appl. Crystallogr. 30 (1997) 565.

8. Farnugia, L. J.: WinGX suite for small-molecule single-crystal crystallography. J. Appl. Crystallogr. 32 (1999) 837-838. 